\title{
A STOCHASTIC CONTROLLED SCHRÖDINGER EQUATION: CONVERGENCE AND ROBUST STABILITY FOR NUMERICAL SOLUTIONS
}

\author{
Cutberto Romero-Meléndez \\ Basic Sciences Department \\ Metropolitan Autonomous University \\ Mexico \\ cutberto@azc.uam.mx
}

\author{
David Castillo-Fernández \\ Department of Applied Mathematics and Systems \\ Metropolitan Autonomous University \\ Mexico \\ dcastillof@cua.uam.mx
}

Article history:

Received 29.08.2021, Accepted 25.11.2021

\begin{abstract}
In this paper we study the stochastic stability of numerical solutions of a stochastic controlled Schrödinger equation. We investigate the boundedness in second moment, the convergence and the stability of the zero solution for this equation, using two new definitions of almost sure exponential robust stability and asymptotic stability, for the Euler-Maruyama numerical scheme. Considering that the diffusion term is controlled, by using the method of Lyapunov functions and the corresponding diffusion operator associated, we apply techniques of X. Mao and A. Tsoi for achieve our task. Finally, we illustrate this method with a problem in $\mathrm{Nu}$ clear Magnetic Resonance (NMR).
\end{abstract}

\section{Key words}

Stochastic Optimal Control, Maximum Stochastic Principle, Euler-Maruyama Scheme.

\section{Introduction}

The stochastic controlled Schrödinger equation depending on time considerate in our study, corresponds to a two-level quantum system describing a spin 1/2particle in a constant and longitudinal static electromagnetic field in the direction of the $\mathrm{Z}$ axis and two randomly time varying electromagnetic fields along the $\mathrm{X}$ axis and $\mathrm{Y}$ axis, respectively. In general terms, that equations is:

$$
\frac{d}{d t} \vec{\psi}(t)=-i H(u(t)) \vec{\psi}(t)
$$

where the state $\vec{\psi}:[0, T] \rightarrow \mathbb{C}^{2}$ is a vector representing the unitary ket $|\psi\rangle, T \in \mathbb{R}$ is the duration of the process, $u:[0, T] \rightarrow \mathbb{R}, u(t)=u_{1}(t)+u_{2}(t)$ is the control and the energy of the system is represented by the Hamiltonian $H(t)$. In [Romero, 2020] we have considered the following stochastic differential equation:

$d \overrightarrow{x_{t}}=\left(S_{z}+u_{y}(t) S_{y}(t)\right) \vec{x}(t) d t+u_{x}(t) S_{x}(t) \vec{x}(t) d W_{t}$

where $\overrightarrow{x_{t}}=\vec{x}(t)$ is obtained splitting real and imaginary parts of $\vec{\psi}, W_{t}=W(t)$ is a standard Wiener process and $S_{z}, S_{y}$ and $S_{x}$ are the Pauli real-splitted matrices. The optimal control problem is the following: to find controls $u_{1}(t), u_{2}(t) \in L^{1}([0, T], \mathbb{R})$ which steers the initial state $\vec{x}(0)$ of the stochastic system (2) to the final state $\vec{x}(T)$ in $\mathbb{C}^{2}$ and minimizes over $0 \leq t \leq T$ the energy cost functional of Bolza type, following:

$J(u)=\mathbb{E}\left(\left\langle x^{\top}(T)|O| x(T)\right\rangle+\int_{0}^{T}\left(u_{1}^{2}(t)+u_{2}^{2}(t)\right) d t\right)$

where $\mathbb{E}(f)$ denotes the conditional expectation with respect to $f, T=\frac{\pi}{\sqrt{2}}$ and $O$ is the observable operator with the target information $O=\vec{x}(T) \vec{x}^{\top}(T)$, which will allow an optimal evolution of the system.

It is known that the system (2) can be solved only through numerical methods. The algorithm used in [Romero, 2020] via the Euler-Maruyama scheme, seeking the numerical solution of the optimal control problem mentioned, is very sensitive to the parameters values related with the NMR phenomenon.

The Euler-Maruyama method is the simplest numerical method for solving stochastic differential equations Let $\|\cdot\|$ be the Euclidean norm in $\mathbb{R}^{n}$ and let's consider the trace as the norm in $\mathbb{R}^{n m}$. Denoting $x_{k}=x\left(t_{k}\right)$, this method computes discrete approximations $x_{k+1}=$ $x_{k}(k \Delta t)$, selecting a grid of $[0, T]: 0<t_{0}<\cdots<$ 
$t_{N}=T$ and satisfying the following stochastic difference equation:

$x_{k+1}=x_{k}+S_{3} x_{k} \Delta t_{k+1}+\left(S_{1} u_{1}(t)+S_{2} u_{2}(t)\right) x_{k} \Delta W_{k+1}$

where

$$
\begin{aligned}
\Delta t_{k+1} & =t_{k+1}-t_{k} \\
\Delta W_{k+1} & =W\left(t_{k+1}\right)-W\left(t_{k}\right)
\end{aligned}
$$

So, we have:

$$
\begin{aligned}
x_{k+1}= & x_{k}+\int_{t_{k}}^{t_{k+1}} S_{3} x_{k} \Delta t_{k+1} \\
& +\int_{t_{k}}^{t_{k+1}}\left(S_{1} u_{1}(t)+S_{2} u_{2}(t)\right) x_{k} \Delta W_{k+1}(5)
\end{aligned}
$$

Each one of these integrals approximate the corresponding integrals of equation (2). In this process, we are interested in the zero solution or equilibrium position of this equation, by using the corresponding Euler-Maruyama numerical scheme in equation (4).

Studying the stability of the numerical schemes for stochastic controlled Schrödinger equations and computing approximations, it's important to analyze the choice of step size $\Delta t_{k}$ in order to extend, by very large time, the stability properties to the exact solution of the corresponding stochastic controlled Schrödinger equation.

In [Burrage, 2000] the study of the stability of stochastic numerical equations of type It is considered, but only stochastic differential equations with multiplicative noise in the linear scalar case are discussed and no control is considered. In [Tocino, 2005] the authors discuss the numerical stability of stochastic differential equations and numerical methods. They use definitions of stochastic stability and asymptotic stochastic stability of an equilibrium position of stochastic differential equations. In our case, we prefer to use the exponential stochastic stability, which is the one used by [Mao, 2015] and [Tsoi, 1997], but we consider variants of the same, more robust and adequate to the model of the controlled stochastic Schr"odinger equation, studied here.

All of the above motivates our interest in investigating the stability and robustness analysis of numerical computations in this model.

\section{Stochastic stability}

\subsection{Almost sure exponential robust stability and asymptotic stability}

In the choice of the optimal control to steer, with probability one, two quantum states for a stochastic quantum system, minimizing the cost, we require some asymptotic stability properties and it is important to have several stability results that allow achieving the desired task. There are different types of exponential stochastic stability [Mao, 1994; Khasminskii, 1981; Tsoi, 1997] and we look for the most suitable for our optimal control problem. Our definitions of almost sure exponential robust stability and asymptotic stability are variants in the control case, of Mao definitions for the stability of numerical schemes, [Mao, 2015]. We will introduce the definitions of almost sure exponential robust stability and asymptotic stability of the trivial solution of the numerical scheme in equation (5), associated to the stochastic controlled Schrödinger equation (2).

First of all, in order to addressing the concepts of stochastic stability and to adapt the classical Lyapunov theory, we introduce a Lyapunov function $V(x, t) \in$ $C^{2,1}\left(\mathbb{R}^{n} \times \mathbb{R}^{+} ; \mathbb{R}^{+}\right)$, with $C^{2,1}\left(\mathbb{R}^{n} \times \mathbb{R}^{+} ; \mathbb{R}^{+}\right)$the family of all non-negative functions $V(x, t)$, continuously twice differentiable in $x$ and once in $t$, defined on $\mathbb{R}^{n} \times \mathbb{R}^{+}$, which allows to guarantee the stability of the solution of the general stochastic differential equation

$$
d x_{t}=b\left(x_{t}, t, u_{t}\right) d t+\sigma\left(x_{t}, t, u_{t}\right) d W_{t}
$$

where, $b: \mathbb{R}^{m} \times \mathbb{R}^{+} \times \mathbb{R} \rightarrow \mathbb{R}^{m} \sigma: \mathbb{R}^{m} \times \mathbb{R}^{+} \times \mathbb{R} \rightarrow$ $\mathbb{R}^{m^{2}}$, and $W(t)$ is a one dimensional Brownian motion. The Lyapunov function $V(x, t)$ must satisfy, according to Itô's formula, the equation:

$$
d V(x, t)=L V(x, t) d t+V_{x}(x, t) \sigma(x, t, u) d W
$$

where $L: \mathbb{R}^{+} \times \mathbb{R}^{n} \rightarrow \mathbb{R}$ is the diffusion operator acting on $V(x, t)$, defined by:

$$
\begin{aligned}
L= & \frac{\partial}{\partial t}+\sum_{i=1}^{n} b(t, x) \frac{\partial}{\partial x_{i}} \\
& +\frac{1}{2} \sum_{i, j=1}^{n}\left(\sigma(x, t, u) \sigma^{\top}(x, t, u)\right)_{i j} \frac{\partial^{2}}{\partial x_{i} \partial x_{j}}
\end{aligned}
$$

It is also convenient to introduce the operator $Q: \mathbb{R}^{+} \times$ $\mathbb{R}^{n} \rightarrow \mathbb{R}$, which, acting on $V(x, t)$, has the following form:

$$
Q V(x, t)=\operatorname{trace}\left[\sigma^{\top}(x, t, u) V_{x}^{\top}(x, t) V_{x}(x, t) \sigma(x, t, u)\right.
$$

Moreover, considering the stochastic differential system (6), we assume the following hypothesis: there are constants $C_{1}, C_{2}, C_{3}$, and $C_{4}$, such that:

(H1) Both $b(x, t, u)$ and $\sigma(x, t, u)$ satisfy the Lipschitz and linear growth conditions, in the $x$ variable:

$$
\begin{aligned}
\|b(x, t, u)-b(y, t, u)\| & \leq C_{1}\|x-y\| \\
\|\sigma(x, t, u)-\sigma(y, t, u)\| & \leq C_{2}\|x-y\|
\end{aligned}
$$

(H2) The controls satisfy the following estimates: $\left\|u_{i}(t)\right\| \leq C_{3}, i=1,2$

(H3) For the numerical solution $x_{0}$ we have following estimates: $E\left\|x_{0}\right\|^{2} \leq C_{4}$

Finally, we introduce the following definitions: 
Definition 1. Let $p \in(0,1]$ be arbitrary, $\Delta t>0$ and $\left\{\lambda_{k}(t)\right\}_{k}$ a positive continuous and increasing sequence. The numerical scheme defined by equation (5) is called almost surely exponentially and robustly stable, if there exists a constant $r>0$, such that:

$$
\lim _{k \rightarrow \infty} \sup \frac{1}{\log \lambda_{k}(\Delta t)} \log \left\|x_{k}\right\|^{p}<-r \quad \text { a.s. }
$$

Definition 2. Let $\Delta t>0$ and $f: \mathbb{R} \rightarrow \mathbb{R}$ a continuous and increasing function. The numerical scheme defined by equation (5) is called asymptotically stable if there exists a constant $l>0$, such that:

$$
\lim _{k \rightarrow \infty} \sup \frac{\log f\left(x_{k}\right)}{k}<-l
$$

Definition 1 is a strong variant of the definition of almost sure exponential stability given in [Tsoi, 1997], which generalizes it in the case where the diffusion term depends on the control, due to the use of $\lambda_{k}(t)$ function. The generalization proposed by Definition 2, with respect to the definition in [Tsoi, 1997], [Mao, 2015] and [Mora, 2017], consists on smoothing the effect of the norm of $x_{k}$, through the use of the function $f$.

\subsection{Main results}

In this section we present the boundedness in second moment, the convergence, the asymptotic stability and the almost sure exponential and robust stability of the zero solution for the Euler-Maruyama numerical scheme, using our definitions.

Theorem 1. Let $x_{k}$ be the numerical solution of the Euler-Maruyama method of system (2). Under assumptions (H1), (H1) and (H3), there exist a positive constant $M_{0}$, such that:

$$
E\left|x_{k}\right|^{2} \leq M_{0}\left|x_{0}\right|^{2}
$$

Proof. let us introduce the processes

$$
Z_{1}(t)=\sum_{k=0}^{\infty} x_{k} 1_{[k \Delta t,(k+1) \Delta t]}(t)
$$

So, using (6):

$x_{k+1}=x_{0}+\int_{0}^{t_{k+1}} b\left(Z_{1}(s)\right) d s+\int_{0}^{t_{k+1}} \sigma\left(Z_{1}(s)\right) d W_{s}$

Hence

$$
\begin{aligned}
\left|x_{k+1}\right| \leq & 3\left|x_{0}\right|^{2}+3\left|\int_{0}^{t_{k+1}} b\left(Z_{1}(s)\right) d s\right|^{2} \\
& +3\left|\int_{0}^{t_{k+1}} \sigma\left(z_{1}(s)\right) d W_{s}\right|^{2}
\end{aligned}
$$

By the linear growth conditions (H1)-(H3), the Holder's inequality and properties of the Itô integral, we deduce that there exists $K$, such that

$$
E\left|x_{k+1}\right|^{2} \leq 3\left[\left|x_{0}\right|^{2}+K^{2}\left(t_{k+1}+1\right) \int_{0}^{t_{k+1}} E\left|z_{1}(s)\right|^{2}\right] d s
$$

Consequently, for $t_{k+1} \leq T$, we get:

$$
E\left|x_{k+1}\right|^{2} \leq 3\left|x_{0}\right|^{2}+3 K(T+1) \Delta t \sum_{j=0}^{k} E\left|x_{j}\right|^{2}
$$

Furthermore, by the Gronwall's inequality, this implies that:

$$
E\left|x_{k+1}\right|^{2} \leq 3\left|x_{0}\right|^{2} e^{3 T K^{2}(T+1)}
$$

Therefore, we conclude:

$$
E\left|x_{k+1}\right|^{2} \leq M_{0}\left|x_{0}\right|^{2}
$$

where $M_{0}=3 e^{3 T K^{2}(T+1)}$.

Theorem 2. Let $x_{k}$ be the numerical solution of the Euler-Maruyama method of system (2) and $X(t)$ its exact solution. Under assumptions (H1)-(H3), for T and $\Delta t$ defined in the Euler-Murayama scheme we have the following estimates, where $P$ is a constant:

$$
E\left|x_{k}-X\left(t_{k}\right)\right| \leq P^{\frac{1}{2}}(\Delta t)^{\frac{1}{2}} e^{(T+1)\left(C_{1}+C_{2}\right)}
$$

Proof. Let's define $Z_{1}(t)=\sum_{k=0}^{\infty} x_{k} 1_{[k \Delta t,(k+1) \Delta t]}(t)$ Using (6) we get, for $\leq t_{k+1} \leq T$ and $x_{0} \neq X(0)$ :

$$
\begin{aligned}
x_{k+1}-X\left(t_{k+1}\right)= & x_{0}-X(0) \\
& +\int_{0}^{t_{k+1}}\left[b\left(Z_{1}(s)\right)-b(X(s))\right] d s \\
& +\int_{0}^{t_{k+1}}\left[\sigma\left(Z_{1}(s)\right)-\sigma(X(s))\right] d W(s)
\end{aligned}
$$

Hence it follows that

$$
\begin{aligned}
E\left|x_{k+1}-X\left(t_{k+1}\right)\right|^{2} \leq & E\left|x_{0}-X(0)\right|^{2} \\
& +E \mid \int_{0}^{t_{k+1}}\left[b\left(Z_{1}(s)\right)-b(X(s))\right]^{2} d s \\
& +\left.\int_{0}^{t_{k+1}}\left[\sigma\left(Z_{1}(s)\right)-\sigma(X(s))\right]\right|^{2} d W(s)
\end{aligned}
$$

We then apply the inequality

$$
|a+b+c|^{2} \leq 3|a|^{2}+3|b|^{2}+3|c|^{2}
$$

and Schwarz's inequality to get:

$$
\begin{aligned}
E\left|x_{k+1}-X\left(t_{k+1}\right)\right|^{2} & \leq 3 \Delta t E\left|x_{0}-X(0)\right|^{2} \\
& +3 E \mid \int_{0}^{t_{k+1}}\left[\left.b\left(Z_{1}(s)-b(X(s))\right] d s\right|^{2}\right. \\
& +3 E \mid \int_{0}^{t]_{k+1}}\left[\left.\sigma\left(Z_{1}(s)-\sigma(X(s))\right] d W(s)\right|^{2}\right.
\end{aligned}
$$


Using Itô's isometry:

$$
\begin{aligned}
& E\left|x_{k+1}-X\left(t_{k+1}\right)\right|^{2} \leq 3 \Delta t E\left|x_{0}-X(0)\right|^{2} \\
& +3 T \int_{0}^{t_{k+1}} E\left|b\left(Z_{1}(s)\right)-b(X(s))\right|^{2} d s P\left[\operatorname { s u p } _ { 0 \leq t \leq T } \left[\int_{0}^{T}\left(\sigma^{\top}(x, t, u), V(x, t)\right) d W(s)\right.\right. \\
& \left.+3 \int_{0}^{t_{k+1}} E\left|\sigma\left(Z_{1}(s)\right)-\sigma(X(s))\right|^{2} d s-\frac{\alpha}{2} \int_{0}^{T} \operatorname{trace}\left[\sigma^{\top}(x, t, u) V(x, t)^{\top} V(x, t) \sigma(x, t, u)\right] d s\right] \\
& >\beta] \leq e^{-\alpha \beta}
\end{aligned}
$$

a Lyapunov function $V(x, t) \in C^{2,1}\left(\mathbb{R}^{n} \times \mathbb{R}^{+} ; \mathbb{R}^{+}\right)$,

$$
\begin{aligned}
E\left|x_{k+1}-X\left(t_{k+1}\right)\right|^{2} & \leq 3 \Delta t E\left|x_{0}-X(0)\right|^{2} \\
& +(3 T+3) \int_{0}^{t_{k+1}}\left(C_{1}+C_{2}\right) E \mid Z_{1}- \\
& \leq 3 \Delta t E\left|x_{0}-X(0)\right|^{2} \\
& +(3 T+3)\left(C_{1}+C_{2}\right) \sum_{j=0}^{k} E \mid x_{j}-X
\end{aligned}
$$

Proof. For the demonstration, see for example [Friedman, 1976].

Theorem 3. Let $p \in(0,1]$ be arbitrary. Assume that the conditions of Lemma 1 are satisfied and let $\left\{x_{k}\right\}_{k}$ a Markov process. If there exists a Lyapunov function

Assuming that the size of the difference between initial conditions is $P:\left|x_{0}-X(0)\right| \leq P$, the discreet Gronwall's inequality implies that:

$$
E\left|x_{k+1}-X\left(t_{k+1}\right)\right| \leq(3 P)^{\frac{1}{2}}(\Delta t)^{\frac{1}{2}} e^{(T+1)\left(C_{1}+C_{2}\right)}
$$

Corollary 1. Assuming the conditions of Theorem 2, the probability $\mathcal{P}$ that the error $\mathcal{E}$ is small, tends to 1 :

$$
\lim _{t \rightarrow \infty} \mathcal{P}\left(\left\|x_{k}-X\left(t_{k}\right)\right\| \leq \Delta_{t}^{\frac{1}{4}}\right) \geq 1
$$

Proof. Defining the error as $\mathcal{E}_{\Delta_{t}}=E\left\|x_{k}-X\left(t_{k}\right)\right\|$, by (12) in the Theorem 2, we have:

$$
\mathcal{E}_{\Delta_{t}} \leq(3 P)^{\frac{1}{2}}(\Delta t)^{\frac{1}{2}} e^{(T+1)\left(C_{1}+C_{2}\right)}
$$

and we know by Markov inequality that:

$$
\begin{gathered}
\mathcal{P}\left(\left\|x_{k}-X\left(t_{k}\right)\right\| \geq \Delta_{t}^{\frac{1}{4}}\right) \leq \frac{\mathcal{E}_{\Delta_{t}}}{\Delta_{t}^{\frac{1}{4}}} \\
\quad \leq(3 P)^{\frac{1}{2}}(\Delta t)^{\frac{1}{2}} e^{(T+1)\left(C_{1}+C_{2}\right)}
\end{gathered}
$$

so, if we put $M=(3 P)^{\frac{1}{2}}(\Delta t)^{\frac{1}{2}} e^{(T+1)\left(C_{1}+C_{2}\right)}$ and we consider the event:

$$
\left\|x_{k}-\left(X t_{k}\right)\right\| \geq \Delta_{t}^{\frac{1}{4}} M
$$

we have:

$$
\mathcal{P}\left(\left\|x_{k}-X\left(t_{k}\right)\right\| \leq \Delta_{t}^{\frac{1}{4}}\right) \geq 1-M \Delta_{t}^{\frac{1}{4}}
$$

By taking $t \rightarrow \infty$, we conclude the expression (13).

Lemma 1 (Exponential martingale inequality). Let $\alpha, \beta$ be any positive numbers. Let $X(t)$ be a solution of the stochastic differential equation (6). If there exists

$X\left(t_{j}()_{0}, t\right) \in C^{2,1}\left(\mathbb{R}^{n} \times \mathbb{R}^{+} ; \mathbb{R}^{+}\right)$, a positive continuous and increasing sequence $\left\{\lambda_{k}(t)\right\}_{k}, \lambda: \mathbb{R} \rightarrow \mathbb{R}^{+}$, with $\lambda_{k}(t) \geq 1$, a continuous and non-negative function $g$ : $\mathbb{R} \rightarrow \mathbb{R}$, such that:

a) $L V(x, t)<-g(t) V(x, t), \forall t \in \mathbb{R}^{+}$

b) $\left\|x_{k}\right\|^{p} \lambda_{k}(t)<V(x, t), \quad \forall t \in \mathbb{R}^{+} \forall k \in \mathbb{N}$

then, the numerical scheme defined in equation (4) is almost surely exponentially and robustly stable.

Proof. We define processes $z_{1}(t)$ and $z_{2}(t)$ as following:

$$
\begin{aligned}
& z_{1}(t)=\sum_{k=0}^{\infty} x_{k} 1_{[k \Delta t,(k+1) \Delta t]}(t) \\
& z_{2}(t)=\sum_{k=0}^{\infty} x_{k+1} 1_{[k \Delta t,(k+1) \Delta t]}(t)
\end{aligned}
$$

for $x_{k}$ satisfying the equation (4). On the other hand, we can use the equation (7) and Ito's formula, to get

$$
\begin{aligned}
d V\left(z_{2}(t), t\right)= & V_{t}\left(z_{2}(t), t\right) d t+V_{x}\left(z_{2}(t), t\right) d z_{2} \\
& +\frac{1}{2} \operatorname{trace}\left[\sigma^{\top}(x, t, u) V_{x x}(x, t) \sigma(x, t, u)\right]
\end{aligned}
$$

The expression:

$$
\begin{aligned}
d \log V\left(z_{2}(t), t\right)= & \frac{1}{V\left(z_{2}(t), t\right)}\left[d V\left(z_{2}(t), t\right)\right. \\
& \left.-\frac{1}{2} \frac{1}{V\left(z_{2}(t), t\right)} d V^{2}\left(z_{2}(t), t\right)\right]
\end{aligned}
$$

implies that:

$$
\begin{aligned}
& \log V\left(z_{2}(t), t\right)=\log V\left(x_{k}, k\right)+ \\
& \int_{0}^{t_{k+1}} \frac{1}{V\left(z_{1}, s\right)}\left[L V\left(z_{1}(s), s\right)-\frac{1}{2} \frac{Q V\left(z_{1}(s), s\right)}{V\left(z_{1}(s), s\right)}\right] d s+ \\
& \int_{0}^{t_{k+1}} \frac{1}{V\left(z_{1}, s\right)} \sum_{k=1}^{n}\left(S_{2} u_{2}\left(t_{k+1}\right)+S_{1} u_{1}\left(t_{k+1}\right)\right) x_{k} 1_{[k \Delta s,(k+1) \Delta}
\end{aligned}
$$


Let's use Lemma 1, taking $T=t_{k+1}, \beta=2 \log C$, $\alpha=1$ and

$\sigma(x, t, u)=\left(S_{2} u_{2}\left(t_{k+1}\right)+S_{1} u_{1}\left(t_{k+1}\right)\right) x_{k} 1_{[k \Delta t,(k+1) \Delta t]}(t)$

to obtain that the probability of the following event $\mathcal{E}$ :
Proof. Let $V(x, t)$ and $f$ as in the hypothesis. Similarly to proof of theorem 3, there exist $r, C$ constants, such that, using equation (16) and hypothesis b), we can write:

$$
\frac{\log V\left(z_{2}(t), t\right)}{k} \leq \frac{1}{k}\left[\log V\left(x_{0}, 0\right)-r t_{k+1}+2 \log C\right]
$$

$$
\begin{aligned}
& \sup _{0 \leq t} \int_{0}^{t_{k+1}}\left(S_{1} u_{1}\left(t_{k+1}\right)+S_{2} u_{2}\left(t_{k+1}\right)\right) x_{k} 1_{[k \Delta t,(k+1) \Delta t]}(t) V_{x}(x, t) d W(s)- \\
& \frac{1}{2} \int_{0}^{T}\left(\left(S_{1} u_{1}\left(t_{k+1}\right)+S_{2} u_{2}\left(t_{k+1}\right)\right) x_{k} 1_{[k \Delta t,(k+1) \Delta t]}(t)\right)^{2} V_{x}^{2} \frac{\log f\left(x_{k}\right)}{(x, t) d_{s}>2} \leq \frac{1}{\log }\left[\log V\left(x_{0}, 0\right)-r t_{k+1}+2 \log C\right]
\end{aligned}
$$

is $P(\mathcal{E}) \leq C^{-2}$ Then, by Borel-Cantelli lemma, there is a random integer $k_{0}$ such that, for all $k \geq k_{0}$, the following inequality is hold:

$$
\begin{aligned}
& \int_{0}^{t_{k+1}}\left(S_{1} u_{1}\left(t_{k+1}\right)+S_{2} u_{2}\left(t_{k+1}\right)\right) x_{k} 1_{[k \Delta t,(k+1) \Delta t]}(t) V_{x}(x, t) d W(s)-\quad \lim _{k \rightarrow \infty} \frac{1}{k} \log f\left(x_{k}\right) \leq-l \\
& \frac{1}{2} \int_{0}^{T}\left(\left(S_{1} u_{1}\left(t_{k+1}\right)+S_{2} u_{2}\left(t_{k+1}\right)\right) x_{k} 1_{[k \Delta t,(k+1) \Delta t]}(t)\right)^{2} \begin{array}{c}
\text { and this implies the conclusion. } \\
V_{x}^{2}(x, t) d s \leq 2 \log C \\
\mathbf{3} \text { Example }
\end{array}
\end{aligned}
$$

\section{Example}

Now, we can use the equation (15) to get:

$$
\begin{aligned}
& \int_{0}^{t_{k+1}} \frac{1}{V\left(z_{1}(s), s\right)} \sum_{k=1}^{n}\left(S_{2} u_{2}\left(t_{k+1}\right)\right. \\
& \left.+S_{1} u_{1}\left(t_{k+1}\right)\right) x_{k} 1_{[k \Delta s,(k+1) \Delta s]} \frac{\partial V}{\partial z_{1}} d W \\
& -\frac{1}{2} \int_{0}^{t_{k+1}} Q V\left(z_{1}(s), s\right) \leq 2 \log C
\end{aligned}
$$

hence, by hypotheses (a) and (b), this turns out:

$\log V\left(z_{2}(t), t\right) \leq \log V\left(x_{0}, 0\right)-\int_{0}^{t_{k+1}} g(t) d t+2 \log C$

Also, from (b), it follows that:

$$
\begin{gathered}
\frac{1}{\log \lambda_{k}(\Delta t)} \log \left\|x_{k}\right\|^{p} \leq \\
\frac{\log V\left(x_{0}, t\right)}{\log \lambda_{k}(\Delta t)}-\frac{\int_{0}^{t_{k+1}} g(t) d t}{\log \lambda_{k}(\Delta t)}+\frac{2 \log C}{\log \lambda_{k}(\Delta t)}
\end{gathered}
$$

Finally, because sequence $\left\{\lambda_{k}(t)\right\}_{k}$ is increasing and $g(t)$ is non-negative, there exists $r>0$, such that

$$
\lim _{k \rightarrow \infty} \sup \frac{1}{\log \lambda_{k}(\Delta t)}\left\|x_{k}\right\|^{p}<-r \text { a.s. }
$$

concluding the proof.

Theorem 4. Let $f: \mathbb{R} \rightarrow \mathbb{R}$ be a function continuous increasing, $\Delta t>0$ and suppose that there exists a Lyapunov function $V(x, t) \in C^{2,1}\left(\mathbb{R}^{n} \times \mathbb{R}^{+} ; \mathbb{R}^{+}\right)$and $a$ constant $r>0$, such that:

a) $f\left(x_{k}\right) \geq V(x, t), \forall t \in \mathbb{R}^{+}, \forall k \in \mathbb{N}$

b) $L V\left(x_{k}\right)<-r V(x, t), \forall t \in \mathbb{R}^{+}$

then, the numerical scheme defined by (4) is asymptotically stable.
By taking $k \rightarrow \infty$, the first and the third term in right hand of (17) tend to 0 and, because $t_{k+1} \geq k+1$, there exist $l>0$, such that:

In this section, we shall discuss an example from NMR to illustrate our theory. We consider the following stochastic optimal control problem, see [Romero, 2020]: To find controls $u_{1}(t), u_{2}(t)$, which steers the initial condition $x(0)=(1,0,0,0)$ of stochastic system (2) to the final state $\left.x\left(\frac{\pi}{\sqrt{2}}\right)=(0,0,0,1)\right)$ minimizing, on the trajectory $x(t)$, the cost functional (3). We will apply Theorem 3 and Theorem 4 to demonstrate the almost sure exponential robust stability and the asymptotic stability of the zero solution of (2), respectively.

Let $M_{1}, M_{2}, M_{3}$ be real constant such that:

$$
\begin{aligned}
u_{1}(t)+u_{2}(t) & \leq M_{1} \\
u_{1}^{\prime}(t)+u_{2}^{\prime}(t) & \leq M_{2} \\
2+4\left(u_{1}^{2}(t)+u_{2}^{2}(t)\right) & \leq M_{1}
\end{aligned}
$$

Let consider $k \in \mathbb{N}$ and $r \in \mathbb{R}$, such that:

$$
r<-\frac{M_{3}}{k M_{2}} M_{1}
$$

We define the following Lyapunov function:

$$
V(x, t)=\left(u_{1}(t)+u_{2}(t)\right)^{r k}\left\|x_{k}(t)\right\|^{2}
$$

Let consider the function $g: \mathbb{R} \rightarrow \mathbb{R}$ given by:

$$
g(t)=-\left(r k \frac{M_{2}}{u_{1}(t)+u_{2}(t)}+M_{3}\right)
$$

and the sequence $\left\{\lambda_{k}(t)\right\}_{k}$ of functions $\lambda_{k}: \mathbb{R} \rightarrow \mathbb{R}^{+}$ defined as:

$$
\lambda_{k}(t)=\left(u_{1}(t)+u_{2}(t)\right)^{r k}
$$



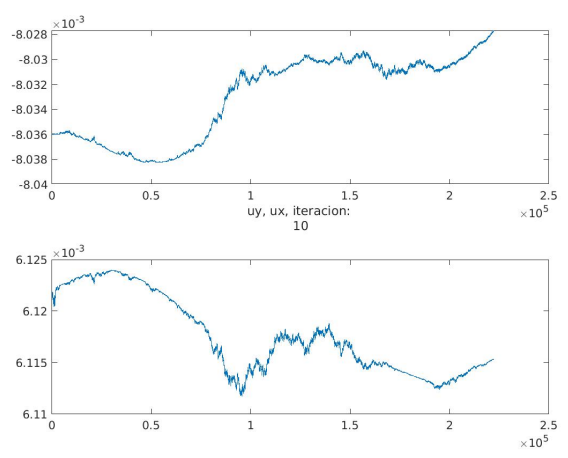

Figure 1. The stochastic optimal controls $u_{1}(t), u_{2}(t)$, obtained in [Romero, 2020], using Euler-Maruyama scheme.

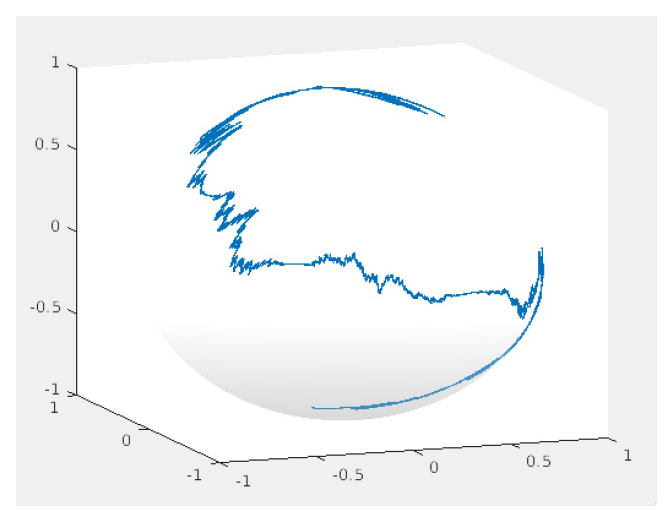

and, for $p \in(0,1]$ :

$$
\left\|x_{k}(t)\right\|^{p} \lambda_{k}(t)<V(x, t), \forall t \in \mathbb{R} \quad \forall k \in \mathbb{N}
$$

Therefore, the conditions of the Theorem 3 are satisfied and we conclude that the solution of the EulerMaruyama numerical scheme defined by equation (2) is almost surely exponentially and robustly stable, for all sufficiently small step sizes $\Delta t_{k}$.

On the other hand, we can use the Lyapunov function $V(x, t)$ given by equation (18) and to take $f(x)=x$ to apply the Theorem 4 to conclude that the solution of the Euler-Maruyama numerical scheme is also asymptotically stable.

Figure 1 shows the optimal stochastic controls obtained in [Romero, 2020], using Euler-Maruyama scheme, where we can see the choice $M_{1}=M_{2}=2$ and $M_{3}=10$ and Figure 2 shows a computer simulation of the optimal stochastic trajectory on the Bloch sphere of the trivial solution $x=\left(x_{1}, x_{2}, x_{3}, x_{4}\right)$ of the numerical scheme in the stochastic controlled Schrödinger equation (2).

\section{Conclusion}

In this paper, we focus on the stability properties of zero solution for a numerical scheme type EulerMaruyama applied to two-level stochastic quantum system. Based on two new definitions for the almost sure exponential robust stability and asymptotic stability, we have demonstrated these kind of stability of the trivial solution for a stochastic controlled Schrödinger equation, generalizing the definitions given in [Tsoi, 1997], [Mao, 2015] and [Mora, 2017], in the case where the diffusion term depends on the control. Our definitions guar-

and consider the controls obtained in [Romero, 2020], given by:

$$
\begin{aligned}
& u_{1}(t)=-\frac{1}{2} \Lambda(t) S_{1} x(t) \\
& u_{2}(t)=-\frac{1}{2} \Lambda(t) S_{2} x(t)
\end{aligned}
$$

where $\Lambda(t)$ is the adjoint state to $x(t)$.

Therefore, in this case, $r<-\frac{10}{k}$.

We compute the corresponding diffusion operator $L V(x, t)$, associated to $V(x, t)$ :

$$
\begin{aligned}
& L V(x, t)= \\
& \left(u_{1}+u_{2}\right)^{r k}\|x\|^{2}\left(r k \frac{u_{1}^{\prime}+u_{2}^{\prime}}{u_{1}+u_{2}}+2+4\left(u_{1}^{2}+u_{2}^{2}\right)\right)
\end{aligned}
$$

Clearly,

$r k \frac{u_{1}^{\prime}+u_{2}^{\prime}}{u_{1}+u_{2}}+2+4\left(u_{1}^{2}+u_{2}^{2}\right) \leq r k \frac{M_{2}}{u_{1}+u_{2}}+M_{3}<0$ antees the robustness of these solutions. We have applied these results in a Nuclear Magnetic Resonance problem, which numerical solution was studied in [Romero, 2020], obtaining almost sure exponential robust stability and asymptotic stability for zero solution of the numerical scheme type Euler-Maruyama.

\section{References}

Berkolaiko, G., Buckwar, E., Conall Kelly, C. and Rodkina, A. (2012). Almost sure asymptotic stability analysis of the Euler-Maruyama method applied to a test system with stabilising and destabilising stochastic perturbations. J. Comput. Math., 15, pp. 71-83.

Burrage, K., Burrage, P. and Mitsui, T. (2000). Numerical solutions of stochastic differential equationsimplementation and stability issues. J. Comput. Appl. Math., 125, pp. 171-182.

Friedman, A. (1976). Stochastic differential equations and applications, V1. Academic Press, New York.

so, we have

$$
L V(x, t)<-g(t) V(x, t), \forall t \in \mathbb{R}
$$


Khasminskii, R. Z. (1981). Stochastic Stability of Differential Equations. Sijthoff and Noordhoff, Alphen aan den Rijn.

Kushner, H. J. (1967). Stochastic Stability and Control. Academic Press, New York.

Mao, X. (1994). Exponential Stability of Stochastic Differential Equations. Marcel Dekker, New York.

Mao, X. (1991). Stability of Stochastic Differential Equations with Respect to Semimartingales. Longman Scientific and Technical, Harlow, UK.

Mao, X. (2015). Almost sure exponential stability in the numerical simulation of stochastic differential equations. SIAM J. Numer. Anal., 53 (1), pp. 370-389.

Mora, C. M., Mardones, H. A., Jiménez, J. C. and Selva,
M. (2017). A stable numerical scheme for stochastic differential equations with multiplicative noise. SIAM J. Numer. Anal., 55 (4), pp. 1614-1649.

Romero-Meléndez, C. and Castillo-Fernández, D. (2020). A numerical approach for the stochastic control of a two-level quantum system. Cybernetics and Physics Journal., 9(7), pp. 107-116.

Tocino, A. (2005). Mean-square stability of secondorder Runge-Kutta methods for stochastic differential equations . Stochastic Analysis and Applications Journal., 15 (4), pp. 643-649.

Tsoi, A. H. and Zhang, B. (1997). Weak exponential stability of stochastic differential equations. J. Comput. Appl. Math., 75, pp. 355-367. 